\title{
One-dimensional modulation of the superconducting boundary condition for thin superconducting films
}

\author{
B. J. Baelus, * B. Partoens, and F. M. Peeters ${ }^{\dagger}$ \\ Departement Fysica, Universiteit Antwerpen, Groenenborgerlaan 171, B-2020 Antwerpen, Belgium
}

(Received 8 February 2006; revised manuscript received 24 April 2006; published 6 June 2006)

\begin{abstract}
Within the framework of the linearized Ginzburg-Landau theory, we study the phase boundary of thin superconducting films. These films are nanostructured such that there is a one-dimensional periodic enhancement of the surface superconductivity which can be realized, e.g., by placing superconducting stripes with a higher critical temperature on top of the film, leading to a one-dimensional modulation of the superconducting boundary condition. We study the influence of this one-dimensional modulation on the enhancement of the critical temperature of the film. In a second step we also place stripes at the bottom of the film and we study both in-phase and out-of-phase modulation.
\end{abstract}

DOI: 10.1103/PhysRevB.73.212503

PACS number(s): 74.20.De, 74.78.-w, 74.62.-c

Due to progress in nanofabrication the study of thin superconducting films and confined mesoscopic samples received a lot of attention during the past decade. The properties of superconductors and therefore their applications are determined by their critical parameters, i.e., the critical field, the critical current, and the critical temperature. By nanostructuring the superconductor, i.e., quantum design, one can modify the properties of an existing superconducting material.

Most studies focus on improving the critical magnetic fields and the critical current. Thin films containing a regular array of pinning centers (see, e.g., Refs. 1-3) are popular study objects. The pinning centers capture the magnetic field, i.e., the vortices, which leads to less dissipation and, hence, an increase of the critical current and critical magnetic field. Also confining the superconducting material to small mesoscopic samples (see, e.g., Refs. 4-6) leads to an enhancement of both the critical current and the critical field. However, this kind of nanostructuring does not lead to an enhancement of the critical temperature.

Fink and Joiner ${ }^{7}$ succeeded in treating the surface of a semi-infinite superconductor half-space by cold working in such a way that the superconductivity near the surface was enhanced, which resulted in a higher critical field and critical current, but also in a higher critical temperature. Another possibility to enhance the critical temperature is to bring the superconductor in contact with a well-chosen superconducting layer with a higher transition temperature. ${ }^{8}$

Few theoretical studies have been performed where superconductivity near the boundary is enhanced leading to an enhancement of the critical temperature. Montevecchi and Indekeu ${ }^{9}$ presented a theoretical study of the effect of confinement on the superconducting/normal transition for systems with surface enhancement. Yampolskii and Peeters ${ }^{10}$ studied thin disks surrounded by a medium which enhances surface superconductivity, and Baelus et al. ${ }^{11}$ studied superconducting cylinders surrounded by such a medium. Recently, Slachmuylders et $a .^{12}$ studied the magnetic field dependence of the critical temperature for superconducting squares with enhanced superconductivity near the boundary.

In the present paper, we study thin nanostructured superconducting films, where surface superconductivity is periodi- cally enhanced. In particular, we consider an infinite superconducting film made of a material with bulk critical temperature $T_{c}$. On top of this film superconducting stripes are placed which are made of a superconducting material with a higher critical temperature. Let us call $d$ the thickness of the film and $W$ the distance between the centers of two stripes. These stripes result in an alternating boundary condition, which we approximate by the function $\beta_{z=d / 2}(x)$ $=\beta_{\max } \sin ^{2}(\pi x / W)$, where $\beta_{\max }=-\xi / b$ with $b<0$ the extrapolation length which determines the boundary condition. ${ }^{13}$ Figure 1 shows schematically the configuration.

Notice that this configuration is well within reach of the possibilities of present day nanofabrication. It presents a route to enhance the critical temperature of superconducting films.

We solve the linearized Ginzburg-Landau equation in the absence of an applied magnetic field

$$
\frac{-\hbar}{2 m} \vec{\nabla}^{2} \Psi=-\alpha(T) \Psi,
$$

where $-\alpha(T)$ equals the energy

$$
E \equiv-\alpha(T)=\frac{\hbar^{2}}{2 m \xi^{2}}\left(1-\frac{T}{T_{c}}\right)
$$

When we express all the lengths in $\xi \equiv \xi(T=0)$ (i.e., $W^{*}$ $=W / \xi$ and $d^{*}=d / \xi$ ) and the energy in $E_{0}=\hbar^{2} / 2 m S$ (i.e., $E^{*}$

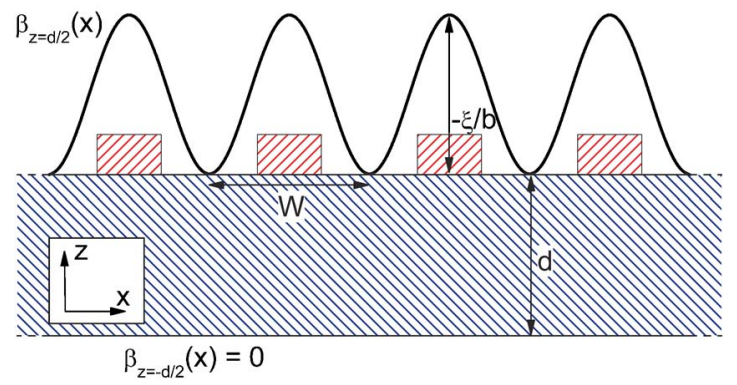

FIG. 1. (Color online) The configuration. An infinite superconducting film (blue area) with thickness $d$ with superconducting stripes (red regions) made of a material with a higher $T_{c}$ on top. The black curve shows our approximation for the boundary condition. 
$\left.=E / E_{0}\right)$ with $S$ the surface area of the cross section of the unit cell, i.e., $S=W d$, then we can rewrite the GinzburgLandau equation as follows:

$$
-\vec{\nabla}^{* 2} \Psi=\varepsilon \Psi
$$

with $\varepsilon \equiv E^{*} / W^{*} d^{*}$ the eigenvalue of the system.

To solve this problem we take into account the general boundary condition in the absence of the magnetic field, i.e.,

$$
-\left.\vec{n} \cdot \vec{\nabla}^{*} \Psi\right|_{\text {boundary }}=\frac{1}{b^{*}} \Psi,
$$

in dimensionless units, with $b^{*}=b / \xi$ the dimensionless extrapolation length.

Since the linearized Ginzburg-Landau equation is only valid at the phase boundary, we know that the temperature $T$ equals the critical temperature $T^{*}$ of the sample, i.e., $T=T^{*}$, and therefore

$$
E=-\alpha\left(T^{*}\right)=\frac{\hbar^{2}}{2 m \xi^{2}}\left(1-\frac{T^{*}}{T_{c}}\right),
$$

where $T_{c}$ is the bulk critical temperature of the film material. In dimensionless units, we find

$$
\varepsilon=\frac{E^{*}}{W^{*} d^{*}}=\left(1-\frac{T^{*}}{T_{c}}\right),
$$

or $T^{*} / T_{c}=-\varepsilon+1$. The critical temperature of the sample $T^{*}$ is obtained from the eigenvalue $\varepsilon$ of the linearized GinzburgLandau equation (3).

From now on, we will express everything in dimensionless units and we will omit the "** except for $T$ * which is the critical temperature of the sample.

We solved Eq. (3) using the finite element technique, on a finite interval $-d / 2<z<d / 2$ ( $d$ is the thickness of the film) and $0<x<W$ (W is the distance between the stripes), with the periodic boundary conditions $\Psi(0, z)=\Psi(W, z), \Psi^{\prime}(x$, $-d / 2) / \Psi(x,-d / 2)=\beta_{z=-d / 2}(x) \quad$ and $\quad \Psi^{\prime}(x, d / 2) / \Psi(x, d / 2)$ $=\beta_{z=d / 2}(x)$.

First, we consider the case where only the upper boundary is modulated, as shown in Fig. 1, i.e., $\beta_{z=-d / 2}(x)=0$ and $\beta_{z=d / 2}(x)=\beta_{\max } \sin ^{2}(\pi x / W)$ where $\beta_{\max }=-\xi / b$ with $b<0$ the extrapolation length which determines the enhancement of the superconductivity near the boundary. When $-\xi / b=0$, i.e., the condition for a normal/insulator boundary, the critical temperature $T^{*}$ equals the bulk critical temperature $T_{c}$, regardless of the thickness of the film or the distance between the stripes. When increasing $-\xi / b$ the Cooper-pair density is locally enhanced at the upper boundary of the film, below the positions of the stripes. As an example, we show in the inset of Fig. 2 the Cooper-pair density for $W=\xi, d=\xi$, and $-\xi / b$ $=0.2$. Near the top of the film, the Cooper-pair density is clearly enhanced due to the presence of the stripe. Notice that in between two stripes, the Cooper-pair density is still enhanced near the upper boundary of the film. Due to this local enhancement of the Cooper-pair density, the critical temperature of the film increases compared to the one of bulk material. In Fig. 2 the critical temperature $T^{*}$ of the superconducting film is given as a function of $-\xi / b$ for $W$

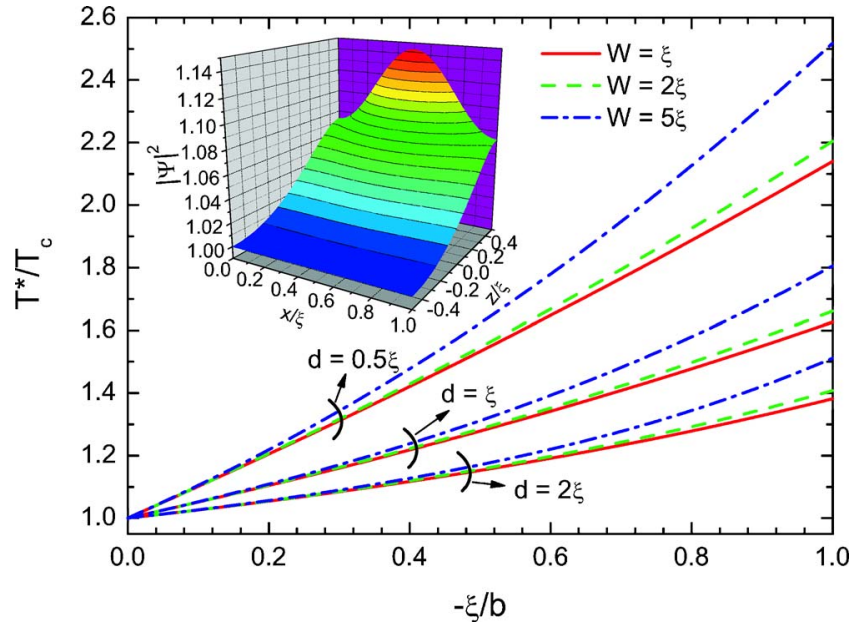

FIG. 2. (Color online) The critical temperature as a function of $-\xi / b$ for a superconducting film with $W=1,2$, and $5 \xi$ and $d=0.5,1$, and $2 \xi$. The inset shows the Cooper-pair density for $W=\xi, d=\xi$, and $-\xi / b=0.2$.

$=1,2$, and $5 \xi$ and $d=0.5,1$, and $2 \xi$. For fixed film thickness $d$ the critical temperature $T^{*}$ enhances when the width of the stripes becomes larger, i.e., increasing $W$. For relative thin

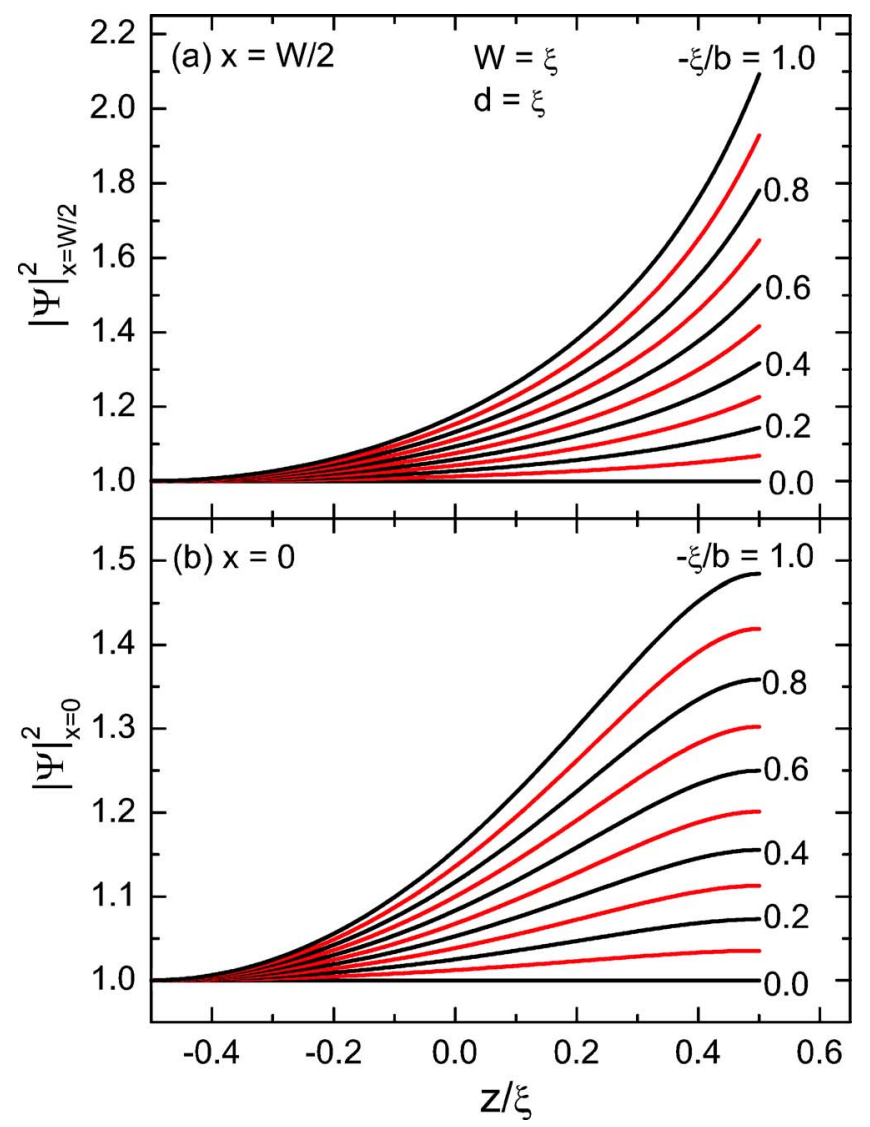

FIG. 3. (Color online) The Cooper-pair density along the $z$ direction (a) for $x=W / 2$, i.e., below the center of the stripe, and (b) for $x=0$, i.e., below the middle between two stripes, for $W=\xi$, $d=\xi$, and different values of $-\xi / b$, i.e., $-\xi / b=0$ up to 1.0 with steps of 0.1 . 


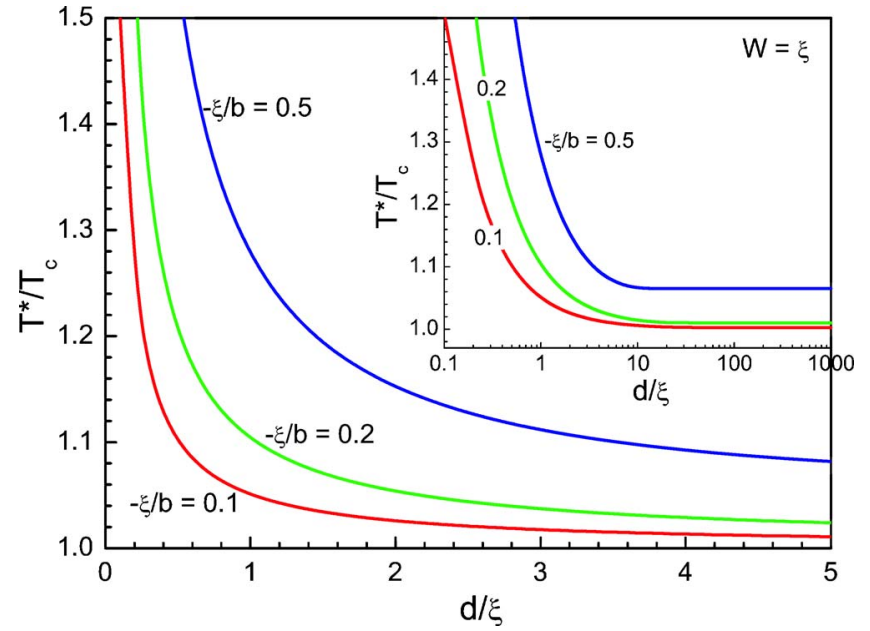

FIG. 4. (Color online) The critical temperature $T^{*}$ as a function of the film thickness for a superconducting film with $W=\xi$ and $-\xi / b=0.1,0.2$, and 0.3 . The inset shows the results for larger thicknesses on a log-scale.

films, i.e., $d \sim \xi$ we find that the dependence of the critical temperature on $d$ is much more pronounced than the dependence on $W$. When decreasing the thickness $d$ of the film the enhancement of the critical temperature $T^{*}$ increases. This can be explained by the fact that a relatively larger part of the film is influenced by the stripes.

Above we already mentioned that near the top of the film the Cooper-pair density is not only enhanced just below the stripes, but also in between two stripes (see the inset of Fig. 2). In Figs. 3(a) and 3(b) we show the enhancement of the Cooper-pair density along the $z$ direction for $x=W / 2$, i.e., below the center of the stripe, and for $x=0$, i.e., below the middle between two stripes, for $W=\xi, d=\xi$, and different values of $-\xi / b$. The Cooper-pair density is always normalized by its value at $z=-d / 2$. With increasing $-\xi / b$ the

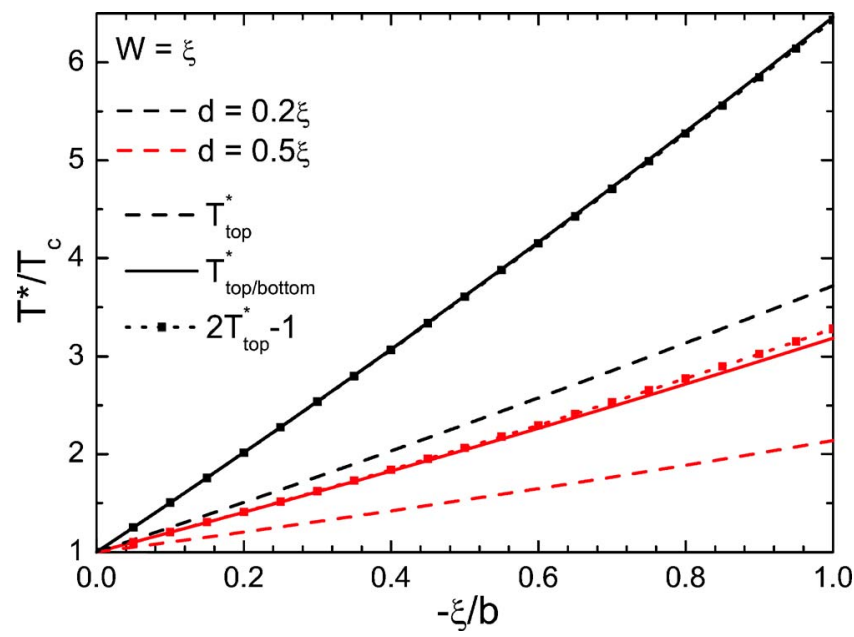

FIG. 5. (Color online) The critical temperature as a function of $-\xi / b$ for a film with thickness $d=0.2$ and $0.5 \xi$ when the stripes are placed at the top only (dashed curves), i.e., $T_{\text {top }}^{*}$, and when the stripes are placed on the top and the bottom (solid curves), i.e., $T_{\text {top/bottom }}^{*}$ The dotted curve with the solid symbols indicates $2 T_{\text {top }}^{*}-1$.
Cooper-pair density enhances in both cases, but the enhancement is much more pronounced below the stripes.

From Fig. 2 it was clear that the critical temperature $T^{*}$ of the film increases, when decreasing the thickness of the film, at least when $d \sim \xi$, because a relatively larger part of the film is influenced by the stripes. What happens when we increase the thickness of the film? When the film is thick, it can be expected that the stripes only influence the Cooperpair density near the top of the film. Figure 4 shows the critical temperature $T^{*}$ of the film as a function of the film thickness for $-\xi / b=0.1,0.2$, and 0.3 . With increasing thickness the critical temperature $T^{*}$ decreases towards a constant value, as is shown in the inset of Fig. 4. For $-\xi / b=0.1,0.2$, and 0.3 we find that the critical temperature becomes $T^{*} / T_{c}$ $=1.003,1.010$, and 1.065 when $d$ becomes sufficiently large.

Up to now, we only placed stripes at the top of the film. Now, we will discuss what happens when we also place stripes at the bottom of the film. We assume that the boundary condition at the bottom of the film is given by $\beta_{z=-d / 2}(x)=\beta_{\text {max }} \sin ^{2}(\pi x / W)$, where $\beta_{\max }=-\xi / b$ and the extrapolation length $b$ has the same value as on the top of the film. First, we will assume that the modulation is the same at the top and the bottom, i.e., in-phase modulation. Later, we will discuss what happens when the stripes at the bottom are shifted over a distance $a$, i.e., $\beta_{z=-d / 2}(x)=\beta_{\text {max }} \sin ^{2}[\pi(x$ $-a) / W]$.

Figure 5 shows the critical temperature as a function of $-\xi / b$ for a film with thickness $d=0.2$ and $0.5 \xi$ when the stripes are placed at the top only (dashed curves), i.e., $T_{\text {top }}^{*}$,

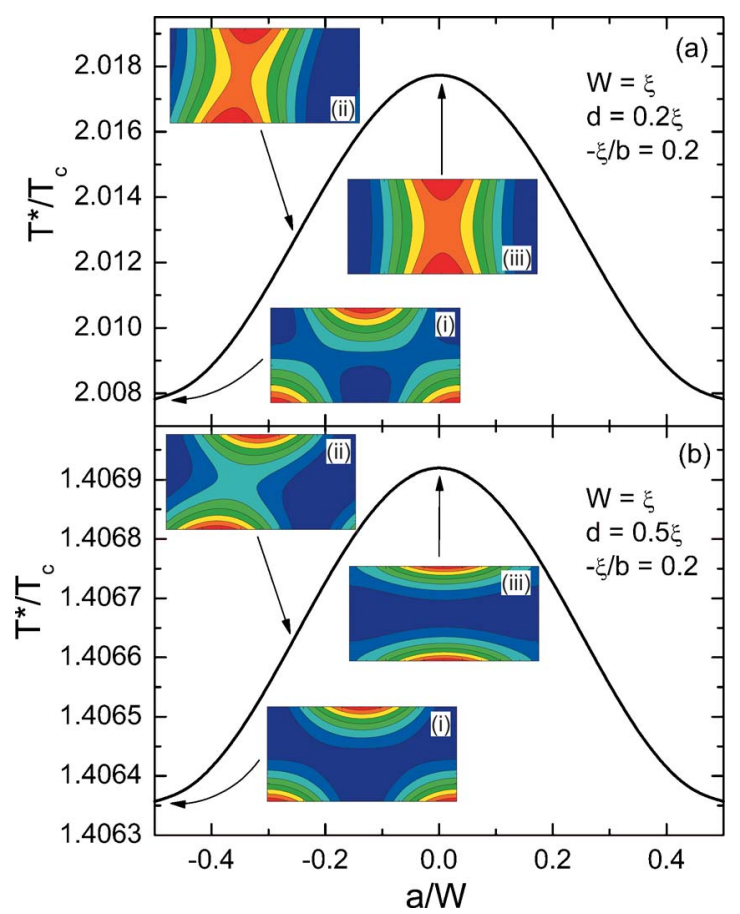

FIG. 6. (Color online) The critical temperature $T^{*}$ as a function of the displacement $a$ of the stripes at the bottom for $W=\xi,-\xi / b$ $=0.2$ and (a) $d=0.2 \xi$ and (b) $d=0.5 \xi$. The insets (i)-(iii) show contour plots of the Cooper-pair density at $a=-W / 2,-W / 4$, and 0 , respectively. High Cooper-pair density is given by red regions, while low Cooper-pair density is given by blue regions. 
and when the stripes are placed on the top and the bottom (solid curves), i.e., $T_{\text {top/bottom }}^{*}$. In both cases the critical temperature obviously increases with increasing $-\xi / b$, but the increase is much stronger when the stripes are placed on both sides. $T_{\text {toplbottom }}^{*}$ can be approached rather closely by $2 T_{\text {top }}^{*}-1$, which is shown by the solid symbols with the dotted curves.

Next, we move the stripes at the bottom over a distance $a$ in the $x$ direction, where $a=0$ means that the stripes at the bottom are at the same $x$ values as the stripes at the top, and $a= \pm W / 2$ means that the stripes at the bottom are just in between the stripes at the top. Figure 6(a) shows the critical temperature $T^{*}$ as a function of the displacement $a$ of the stripes at the bottom for $W=\xi, d=0.2 \xi$, and $-\xi / b=0.2$. The insets (i)-(iii) show contour plots of the Cooper-pair density at $a=-W / 2,-W / 4$, and 0 , respectively. High Cooper-pair density is given by red regions, while low Cooper-pair density is given by blue regions. The critical temperature has a maximum at $a=0$, when the modulation is in-phase, and a minimum when the modulation is out of phase, i.e., $a= \pm W / 2$. Figure 6(b) shows the same but now for a film with thickness $d=0.5 \xi$. For $d=0.2 \xi$ we find that the difference in critical temperature is about $0.5 \%$, while for $d$ $=0.5 \xi$ it is $0.4 \%$. When we further increase the film thickness, the influence of the shift $a$ decreases, because the overlap between the regions of enhancement of superconductivity decreases.

In conclusion, we investigated the one-dimensional modulation of the superconducting boundary condition for thin superconducting films, within the framework of the linearized Ginzburg-Landau theory. For a thin film with a spatial one-dimensional modulation of the boundary condition, we find an increase of the critical temperature with $-\xi / b$ and $W$ and a decrease with $d$. For a modulation of the boundary condition at the top and the bottom we found a maximum critical temperature when both modulations are in-phase.

This work was supported by the Flemish Science Foundation (FWO-Vl), the Belgian Science policy, and the ESFADQJJ program. B.J.B. and B.P. acknowledge support from FWO-Vl. Discussions with J. O. Indekeu, C. Giuraniuc, and A. Slachmuylders are gratefully acknowledged.
*Email address: ben.baelus@ua.ac.be

†Email address: francois.peeters@ua.ac.be

${ }^{1}$ M. Baert, V. V. Metlushko, R. Jonckheere, V. V. Moshchalkov, and Y. Bruynseraede, Phys. Rev. Lett. 74, 3269 (1995).

${ }^{2}$ M. J. Van Bael, K. Temst, V. V. Moshchalkov, and Y. Bruynseraede, Phys. Rev. B 59, 14674 (1999).

${ }^{3}$ M. V. Milosevic and F. M. Peeters, Phys. Rev. Lett. 93, 267006 (2004).

${ }^{4}$ A. K. Geim, I. V. Grigorieva, S. V. Dubonos, J. G. S. Lok, J. C. Maan, A. E. Filippov, and F. M. Peeters, Nature (London) 390, 259 (1997).

${ }^{5}$ V. A. Schweigert, F. M. Peeters, and P. S. Deo, Phys. Rev. Lett. 81, 2783 (1998).

${ }^{6}$ L. F. Chibotaru, A. Ceulemans, V. Bruyndoncx, and V. V. Moshchalkov, Nature (London) 408, 833 (2000); ibid. 86, 1323 (2001).
${ }^{7}$ H. J. Fink and W. C. H. Joiner, Phys. Rev. Lett. 23, 120 (1969).

${ }^{8}$ J. O. Indekeu, F. Clarysse, and E. Montevecchi, in $\mathrm{High} \mathrm{T}_{c} \mathrm{Su}$ perconductors and Related Materials, edited by S.-L. Drechsler and T. Mishonov (Kluwer Academic Publishers, Dordrecht, 2001), pp. 487-504.

${ }^{9}$ E. Montevecchi and J. O. Indekeu, Phys. Rev. B 62, 14359 (2000); Europhys. Lett. 51, 661 (2000).

${ }^{10}$ S. V. Yampolskii and F. M. Peeters, Phys. Rev. B 62, 9663 (2000).

${ }^{11}$ B. J. Baelus, S. V. Yampolskii, F. M. Peeters, E. Montevecchi, and J. O. Indekeu, Phys. Rev. B 65, 024510 (2001).

${ }^{12}$ A. F. Slachmuylders, B. Partoens, and F. M. Peeters, Phys. Rev. B 71, 245405 (2005).

${ }^{13}$ P. G. de Gennes, Superconductivity of Metals and Alloys (Addison-Wesley, New York, 1994). 